\title{
Los autogolpes en América Latina. El caso de Guatemala (1993)
}

\author{
Alfredo Brino Bologna
}

La década del noventa mostró un mapa inédito para América Latina, donde la casi totalidad de los gobiernos del área habían llegado al poder mediante elecciones democráticas. Sin embargo, también se pudo apreciar que asomaron golpes de Estado -algunos de ellos abortados, pero otros que lograron consolidarse en el poder-cuya característica peculiar fue lo que se denominó autogolpe o golpe cívico-militar. Esta nueva forma de inestabilidad política, a pesar de tener antecedentes en la región, se concretó en Perú en 1992. En el presente trabajo se trata de establecer las características del autogolpe de Guatemala en 1993, analizando los factores endógenos y exógenos que impidieron su concreción. La conflictiva relación de poderes en algunos países del área hace necesaria la recurrencia a las enseñanzas que nos dejó el caso guatemalteco para tratar de establecer a partir de ello parámetros de comportamiento democrático que impidan la repetición de una situación como la mencionada.

\section{Introducción.}

Luego de muchos años de golpes de Estado, la década del noventa mostró un mapa inédito de América Latina en el cual se podía detectar que la inmensa mayoría de los gobiemos habían llegado al poder por medio de elecciones democráticas.

Este sintoma alentador de la región se vio alterado con el surgimiento de golpes de Estado en Haití, 1991; Venezuela en dos oportunidades, febrero y noviembre de 1992; Perú, 1992; y Guatemala, 1993.

En un trabajo anterior analicé los casos de Haití, Venezuela y Perú, señalando como conclusión que en los dos primeros se trataba del típico 
golpe latinoamericano, en cambio el de Perú se caracterizaba como un autogolpe o golpe cívico-militar. ${ }^{1}$

El golpe de Estado en Perú, marcaba para la región una tendencia que podía ser imitada, como de hecho se repitió en Guatemala en 1993.

No puede dejar de mencionarse la transición del clásico golpe de Estado latinoamericano hacia este nuevo modelo de autogolpe. En Haití, el gobierno militar nombró el 11 de mayo de 1994 al Presidente de la Corte Suprema de Justicia, Emile Jonassaint, como Presidente provisional hasta el llamado a elecciones para elegir nuevo Presidente, cuyo mandato comenzaría el 7 de febrero de 1995.

La conflictiva relación de poderes en algunos paises del área, hace necesario la recurrencia a las enseñanzas que nos deja el caso guatemalteco para tratar de establecer a partir de ello parámetros de comportamiento democrático que impidan la repetición de una situación como la mencionada.

\section{I.- La situación política, económica y social previa al autogolpe.}

Serrano había asumido la presidencia el 14 de enero de 1991, siendo el segundo civil en ese cargo luego de intermitentes gobiernos democráti$\cos ^{2}$

La situación politica, económica y social de Guatemala en los días previos al golpe estaban demostrando la incapacidad del gobierno de Serrano para controlar la crisis existente. Entre los aspectos más destacados de ese ambiente se pueden mencionar: la existencia de un gobierno con minoria parlamentaria, un clima de agitación estudiantil, resistencia de la población al aumento de las tarifas eléctricas y el estancamiento en las negociaciones con el movimiento guerrillero.

\footnotetext{
${ }^{1}$ El presente trabajo es parte de un estudio más completo donde se tratarả de observar en particular los factores endógenos y exógenos que impidieron la concreción del autogolpe de Guatemala. Si bien en ocasiones se hace difuso separar los dos ámbitos del linkage, creo que se puede trabajar sobre esas diferencias e influencias "intermésticas". A los anteriores golpes de Estado de la presente década se hizo referencia en "La democracia y la Organización de Estados Americanos", Revista Relaciones Internacionales, Instituto de Relaciones Internacionales, Universidad Nacional de La Plata, La Plata, noviembre de 1993, No5, p. 17.

${ }^{2}$ El antecesor de Serrano en el cargo fue Marco Vinicio Cerezo, del partido Democracia Cristiana Guatemalteca, quién gobernó entre 1986 y 1991 , debiendo soportar durante su gestión dos intentos de golpes de Estado, el 11 de mayo de 1988 y el 9 de mayo de 1989.
} 


\section{Gobierno con minoría política en el Congreso Nacional.}

La llegada al poder de Serrano tiene algunas características importantes que es necesario remarcar para poder entender los acontecimientos de 1993.

En primer lugar, debe consignarse que llegó a la presidencia después de efectuarse una segunda. vuelta electoral. De los doce candidatos presentados en las elecciones del 11 de noviembre de 1990, ninguno de ellos obtuvo mayoría absoluta, por lo cual se debía realizar una segunda eiección o ballotage.

Los candidatos más votados fueron: Jorge Carpio Nicolle de la Unión del Centro Nacional (U.C.N.), con el 25,7\%, y Jorge Serrano Elías del Movimiento de Acción Solidaria (M.A.S.), con el 24,1\% de los votos. .

Esta elección tuvo la particularidad de que el partido de la Democracia Cristiana Guatemalteca (D.C.G.), cuyo candidato a la presidencia era Alfonso Cabrera, se ubicara en segundo lugar en la elección de cargos legislativos, logrando alcanzar 28 diputados. La U.C.N. tuvo 41 y el M.A.S. sólo 18.

La elección del 11 de noviembre de 1990 marcó a fuego la gestión de Serrano, ya que sólo obtuvo 18 diputados sobre un total de 115 legisladores.

El 6 de enero de 1991 se realiza la segunda vuelta en la cual triunfa Jorge Antonio Serrano Elías con el $68 \%$ de los votos, sobre Jorge Carpio Nicolle que sólo alcanzó el $32 \% .^{3}$

La realidad política de la elección del 11 de noviembre de 1990, llevó a Serrano a constituir un gobierno de "Unidad Nacional" a través de la alianza con algunos partidos minoritarios. Algunos e estos partidos,

\footnotetext{
${ }^{3}$ Jorge Serrano Elias, un evangelista en un pais en el cual e1 $90 \%$ de la población es católica, atribuyó su victoria a la ayuda de Dios y afirmó que esperaba contar con la ayuda divina durante su gestión. Expresó: "No estamos solos, Dios estä con nosotros. El nos ayudara a movernos en la dirección" correcta". Para, algunos analistas políticos, el éxito electoral en la segunda vuelta se debió a las: decenas de votos de los evangélicos que en un principio votaron por Efrain Rios Mont y que, al ser vetado por los tribunales como candidato por su vinculación a los pasados golpes militares, finalmente optaron votar por Serrano. Es indudable que la elección de Serrano había sorprendido a los observadores politicos. Sin embargo no coincido en calificar la llegada al poder de Serrano como la de un "antipolitico". Eduardo Ulibarri, "Golpes y deslices de Outsiders", Vision, Buenos Aires, 15 de julio de 1993. Es por ello que creo necesario mencionar la actividad pública de Serrano, que si bien muestra una personalidad conflictiva, no por ello debo dejar de reconocer el haber desempeñado y actuado en la política desđe su juventud, característica esta que lo diferencia de Alberto Fujimori y Mario Vargas Llosa. Para ampliar el tema de la actividad politica desarrollada por Serrano mucho antes de su presidencia se puede consultar el diario La Nación de Buenos Aires, del 26 de mayo de 1993. Cable de la agencia EFE, cuyo título es "Un flamante cultor del estilo Fujimori y' Carmen Escribano de León, Tras los pasos de Fujimori"; y "El presidente Serrano quiso todo el poder", Visión, Buenos Aires, 15 de julio de 1994.
} 
como por ejemplo el Partido de Avanzada Nacional (P.A.N.), abandonó el gobierno a los ocho meses de la alianza.

Al concluir su primer año de gestión, se podía observar que tanto la Junta Directiva de la República como la Corte Suprema de Justicia estaban bajo el control de los partidos mayoritarios.

De tal manera que Serrano llegó a 1993 con la ruptura del consenso político que había logrado en 1991, sin posibilidad de negociación para poder reconstituirlo.

Por ello Escribano de León estima que el principal déficit de Serrano al llegar al gobiemo fue la carencia de programa y la necesidad de realizar todo tipo de alianzas en el Congreso, dado que no poseía mayoría propia. La corrupción, los sobornos a la prensa y una decidida vocación hegemónica, fueron las características más sobresalientes del periodo en que gobernó. 4

Esta situación de desequilibrio entre el poder ejecutivo y el Congreso Nacional puede considerarse como una de las causas del golpe de Estado.

\section{Agitación estudiantil.}

Un clima de agitación callejera comenzó en los primeros días de mayo. Los estudiantes tuvieron una activa participación en los mismos.

El origen del malestar se inició a partir de una disposición del Ministerio de Educación por la cual los estudiantes de nivel medio debían reemplazar el "bono de transporte" por un "cédula estudiantil", que de acuerdo a las autoridades gubernamentales permitia a los estudiantes viajar en forma completamente gratuita. El bono no cubría la totalidad del pasaje sino una parte.

Los estudiantes aglutinados en la Asociación de Estudiantes del Nivel Medio (A.N.E.M.) interpretaron que la "cédula estudiantil" representaba una forma velada de mantener mecanismos de control sobre lapoblación estudiantil, manifestando el temor de que este hecho fuera parte de una estrategia de trasfondo militar.

Las manifestaciones estudiantiles avanzaron con la toma de establecimientos públicos y barricadas en las calles de Guatemala.

\footnotetext{
${ }^{4}$ A principios de 1992 se produce una renuncia de quien ejercía el Ministerio de Relaciones Exteriores por el P.A.N., Alvaro Arzú lrigoyen, por "desavenencias" con el Presidente Serrano en el manejo y traiamiento de la política exterior, particularmente por el reconocimiento a la soberanía del territorio de Belice. Este tema, fue motivo de largas disputas entre el Reino Unido y Guatemala. "Panorama Centroamericano/Recorte Político", Boletin del Instituto Centroamericano de Estudios Politicos (INCEP), Guatemala, febrero de 1992. p. 7. Salvo indicación expresa, la bibliografia básica para la elaboración del presente trabajo se extrajo de la mencionada publicación mensual.
} 
El 13 de mayo un estudiante, Abner Hernández Orellana; fue muerto por el disparo de un guardaespaldas del diputado del Partido Revolucionario, Obdulio Chinchilla Vega.

A partir de entonces se producen enfrentamientos más violentos entre los estudiantes y la policía. El diálogo iniciado entre la Ministra de Educación, María Luisa Beltranena de Padilla; y los estudiantes no pudo superar el conflicto.

La Asociación de Estudiantes Universitarios de la Universidad San Carlos (USAC) acompañó las demandas de los estudiantes de nivel medio.

Las barricadas, bombas molotov y quema de omnibuses enfrentó ä la policía con los estudiantes. El agravamiento de la situación trajo comio consecuencia la paralización del transporte.

Frente a esta situación de caos, el Presidente Serrano anunció la intención de implantar el estado de excepción y suspender las garantías constitucionales. La posible aplicación fue cuestionada por gran parte de la población y esto motivó el anuncio de Serrano el 19 de mayo por el cual expresó que no se aplicaría, pero que se implementaban fuertes operativos en toda la ciudad para poner fin a los disturbios.

\section{Aumento de las tarifas de electricidad.}

La propuesta de Serrano de establecer un aumento a las tarifas de electricidad, lo enfrentó al Congreso Nacional que manifestó su disconformidad con la medida.

Un hecho de violencia complicaba aún más la situación social. El 18 de mayo se produce un atentado contra el Diputado del Partido Revolucionario, Obdulio Chinchilla Vega, en el cual perdió la vida uno de los. empleados de la legislatura, resultó herido uno de los guardaespaldas del diputado y el mismo legislador fue internado en un hospital por haber recibido varios impactos de bala.

El diputado Chinchilla Vega se oponía, dentro del Parlamento, a la aprobación de un programa de aumentos al precio del servicio de energía eléctrica enviada por el poder ejecutivo.

El tema del aumento de tarifas eléctricas permaneció invariable, sin lograrse una solución negociada, entre los dos poderes mencionados.

\section{Las negociaciones con el movimiento guerrillero.}

Si bien durante el gobiemo de Serrano se prosiguió la ronda de negociaciones iniciadas por el Presidente Marco Vinicio Cerezo con el movi- 
miento guerrillero Unión Revolucionaria Nacional Guatemalteca (U.R.N.G.), la undécima reunión realizada en México concluyó el 9 de mayo de 1993, sin fijar fecha para un nuevo encuentro.

La ronda de negociaciones se vio perjudicada por la publicación el $1^{\circ}$ de mayo de 1993 de un documento de carácter confidencial.

Luego de este acontecimiento, el representante de la Iglesia Católica, en la Comisión para la Paz (COPAZ), monseñor Quezada Toruño, renunció.

Como se podrá observar, en el mes de mayo de 1993 se conjugaron y agudizaron los conflictos existentes que estaban adormecidos en la sociedad.

Una crítica a la situación imperante en 1993, al Presidente, ministros, justicia, Congreso y Ejército, la describe con aguda crudeza, Jonas, quien concluye que la situación previa al autogolpe era un escenario de ruinas políticas con síntomas de vida incipiente como la Procaduria de Derechos Humanos. 5

De cualquier manera, por una parte lo que se podía observar era la sociedad que estaba gestando nuevas formas de participación y, por la otra, un gobiemo sin capacidad de negociación, respuesta y reacción frente a estos nuevos desafios.

\section{II.- EI autogolpe de Serrano.}

A pesar de la conflictiva situación socioeconómica por la cual estaba atravesando Guatemala en 1993, el Presidente Jorge Serrano Elías aún confiaba en el apoyo popular.

Los signos de esa percepción errónea del Presidente Serrano se fundamentaban en dos acontecimientos políticos.

Por una parte, las elecciones municipales efectuadas el 9 de mayo de 1993, mostraban un porcentaje del 37,7\% del electorado en favor del partido gobernante, tres veces más que los votos obtenidos por el partido opositor más cercano que fue la Democracia Cristiana Guatemalteca. (D.C.G.)

La lectura realizada por los partidos políticos no concordaba con la efectuada por el Presidente. La abstención del $70 \%$ del electorado estaba

\footnotetext{
5 Jonas, Susanne, "Guatemala, El problema democrático", Nueva Sociedad, Caracas, marzo-abril $1994, N^{\circ} 130$, p. 17.
} 
mostrando el desencanto y decepción que expresaba el pueblo con relación a la gestión del gobiemo de Serrano. ${ }^{6}$

El otro hecho contabilizado como favorable, de acuerdo al Presidente, era el desprestigio del Congreso Nacional, de la justicia y de los partidos políticos ante la opinión pública.

Tomando como determinantes los hechos enunciados, el Presidente Serrano anuncia en un mensaje a la nación, el 25 de mayo de 1993, que por decreto resuelve la disolución del Congreso, deja sin efecto la integración de la Corte Suprema de Justicia y de la Corte de Constitucionalidad, remueve de su cargo al Procurador General de la Nación y Jefe del Ministerio Público, y se interrumpe temporalmente la vigencia de 46 artículos de la Constitución Nacional. ${ }^{7}$

En su mensaje a la nación expresó que entregará la presidencia de la República el dia 14 de enero de 1996, tal como lo establece el período constitucional.

Solicita al Tribunal Supremo Electoral que proceda a convocar en sesenta días a una Asamblea Nacional Constituyente que tendrá como objetivo modificar la Constitución Nacional.

En este discurso el Presidente Serrano manifiesta cuáles son las causas que lo llevaron a tomar esta resolución:

"Lo único que a mí me mueve para tomar esta decisión trascendente e importante, es poder garantizar que nuestra Guatemala no sea presa del narcotráfico; que nuestro país no sea un jardín para las mafias, que nuestro país tenga un destino sin corrupción, sin banalidad, sin demagogia".

El mismo día, la Corte de Constitucionalidad hace conocer una resolución por la cual declara "inconstitucional el decreto que contiene las 'Normas Temporales de Gobiemo' emitido por el Presidente de la República con fecha 25 de mayo de 1993, disposiciones que quedan sin vigencia y dejan de surtir efecto".

A pesar de la oposición de la población guatemalteca que se comenzó a manifestar desde el mismo día 25 de mayo y la repercusión desfavorable de la comunidad intemacional, Serrano nombra el 27 de mayo como

\footnotetext{
${ }^{6}$ Sobre las elecciones mencionadas se puede consultar "Panorama Centroamericano/Recorte Politico", Boletin del Instituto Centroamericano de Estudios Politicos (INCEP), Guatemala, Año XXIV (Tercera época), mayo de 1993, N82, p. 7 .

${ }^{7}$ Simultáneamente con la alocución del Presidente Serrano se dio a publicidad el documento "Acuerdo de normas temporales de Gobierno emitido por el Presidente de la República de Guatemala", que lleva la firma de Serrano y del Ministro de Gobernación, Francisco Rolando Perdomo Sandoval.
} 
Presidenta de la Corte Suprema de Justicia a Maria Luisa Beltranera, quien había ocupado anteriormente el Ministerio de Educación.

Al día siguiente el gobierno suspendió a úlitimo momento y sin dar explicaciones una manifestación de apoyo a Serrano. Rigoberta Menchú, Premio Nobel de la Paz, afirmó que el gobierno había destinado 500.000 dólares para organizar el traslado de campesinos para que participen en la marcha.

A medida que pasaban los dias, era cada vez más evidente la pérdida de poder de Serrano. Factores endógenos y exógenos influirían para que en las postrimerías del mes de mayo Serrano intentará otras variables para permanecer en el poder.

El día 29 de mayo, Serrano convoca a una reunión a los embajadores acreditados en Guatemala. En esa ocasión expresó que no era dictador. "Quiero la democracia, pero me vi obligado a hacer esto porque estaba siendo víctima de un plan desestabilizador orquestado por opositores, por la guerrilla y por el narcotráfico. Manifestó que el Presidente de la Corte Suprema de Justicia, Juan José Pocil Peralta, lo quiso chantajear. Según Serrano le dijo: "Si no me das una partida presupuestaria extra ya vas a ver quién soy yo". Expresó que dos diputados le pidieron 30 millones de dólares para aprobar la privatización de la red de energía eléctrica. Denunció que el narcotráfico habia comprado a la Corte Suprema de Justicia Según el Presidente, cada vez que el gobiemo detenía a un narcotraficante ella lo liberaba. Inclusive liberaron a uno de ellos que habian encontrado con una carga de 100 kilogramos de cocaína.

Expresó que los militares estaban muy nerviosos por la ola de intranquilidad y de revueltas en las que había infiltrado guerrilleros para provocar la anarquía. Los militares se estaban cansando de todo y Serrano. los convenció para dar el autogolpe considerando que era lo mejor.

También se expresó desfavorablemente del papel de la prensa que hizo, según Serrano, una campaña brutal contra su gobierno, y desmintió que ahora la censura sea total: "yo les dije a los directores de los diarios que ellos ejercieran su propio control así no nos veíamos obligados a censurarnos". Manifestó que Jorge Carpio, director del matutino El Gráfico y Presidente del partido Unión de Centro Nacional le dijo: "Prefiero que nos censuren ustedes porque en una de esas no leemos bien todo el diario y se nos escapa algo".

Los diplomáticos coincidieron en afimar que en esta ocasión Serrano se mostró con una imagen humilde y conciliadora, que contras- 
taba con el hombre seguro, autoritario y arrogante de los primeros días del golpe de Estado. ${ }^{8}$

En la reunión, Serrano leyó un acta de cuatro puntos que según dijo, estaba negociando con políticos de la oposición. En el texto se preveía el reconocimiento de la crisis de gobernabilidad producida en Guatemala; aceptación opositora de que la remoción de la Corte Suprema de Justicia era irreversible; la formación de un comisión mixta para que, en no más de treinta días, presente una reforma de consenso a la Constitución de 1985; la renuncia de los legisladores a sus bancas y la convocatoria a un plebiscito constituyente y a elecciones legislativas en 60 días adicionales.

Según Serrano, si el acta mencionada era ratificada por la oposición, las garantías constitucionales suspendidas, serían restauradas en ocho o diez días. ${ }^{9}$

Los rumores existentes en la población sobre su posible renuncia, hacen desmentir a Serrano la versión de su dimisión en un mensaje televisivo que se emite el día 31 de mayo.

En el mismo expresó que en ningún momento renunciaría, argumentando que sus medidas son la única vía posible para la consolidación de una verdadera democracia. Aseguraba también que la economía nacional seguía su normal desarrollo, al igual que las relaciones comerciales y la asistencia externa con otros países del mundo.

Ante la aceleración del proceso, en la madrugada del día primero de junio, Serrano convoca en una reunión a un centenar de diputados y dirigentes partidarios tratando de evitar su destitución. Algunos de los asistentes dijeron que les había ofrecido hasta treinta mil dólares y puestos en el gabinete y en la administración pública para comprar su voluntad. ${ }^{10}$

Juan Francisco Reyes López denunció que Serrano le ofreció 150 mil quetzales a cada diputado, a condición de que le brindasen su apoyo a un proyecto de amnistía política.

Ante la presión ejercida por distintos sectores sociales y como consecuencia del cumplimiento por parte de las fuerzas armadas de la sentencia de la Corte de Constitucionalidad del 25 de mayo, Serrano

\footnotetext{
${ }^{8}$ Roberis, Carlos Reymundo, "Serrano, debilitado por las presiones apuraria el retorno a la democracia", La Nación, Buenos Aires, 30 de mayo de 1993. En esta reunión llegó a reconocer ante la sorpresa de los embajadores que se habja comprado un Mercedes Benz de lujo, valuado en 200.000 dólares. Y Serrano preguntó: "¿Qué le ven de extraño que una persona se compre un auto?".

${ }^{9}$ Cardoso, Oscar R., "Serrano propone un plan politico", Clarin, Buenos Aires, 30 de mayo de 1993.

${ }^{10}$ Roberts, Carlos Reymundo, "Victina de su propia guillotina", La Nación, Buenos Aires, 2 de junio de 1993.
} 
abandona la Jefatura de Gobiemo el primero de junio de 1993. ${ }^{11}$ Serrano no renunció al cargo y oficialmente se precisó que hacía abandono del mismo, cuando aún no había cumplido una semana en el gobiemo de facto.

Algunos periodistas afirmaban que ya nadie discutía que la segunda experiencia autogolpista de América Latina había concluido definitivamente.

Sin embargo, debe apreciarse que en estos momentos se comienza a gestar el origen de un segundo autogolpe.

\section{Segundo autogolpe.}

En un comunicado del 31 de mayo, el ejército había expresado que "al haber abandonado el cargo de Presidente de la República, (Serrano), lo" asume temporalmente, como corresponde, de acuerdo con la Constitución, el Vicepresidente de la República, señor Gustavo Adolfo Espina Salguero, quien ha denunciado".

A pesar de la claridad de este comunicado, el Vicepresidente Gustavo Espina, se autoproclama el 2 de junio de 1993, Presidente de la República de Guatemala.

Para respaldar jurídicamente el cargo, Espina intenta que su decisión sea refrendada por el Congreso Nacional. A esos efectos, se realiza una reunión con la asistencia de diputados del M.A.S. y algunos legisladores independientes que respaldaron a Serrano. Esta propuesta no fue apoyada por el Congreso ya que no se logró el quórum necesario.

La Instancia Nacional de Consenso (I.N.C.) inicia acciones judiciales por intermedio del Procurador de los Derechos Humanosy del Ministerio Público, desconociendo al nuevo Jefe de Gobierno y solicita su enjuiciamiento.

El Ministerio Público presenta una solicitud de antejuicio contra Serrano y Espina, exigiendo la extradición del ex-mandatario al gobierno de El Salvador.

El Ministro de Defensa, nombrado por el Presidente Serrano y que permanecía en el cargo, José Domingo García Samoyoa, hace público su respaldo al Vicepresidente Espina.

Espina también logra el respaldo de la Corte Suprema de Justicia, cuyo Presidente Juan José Rodil Peralta, anuncia que apoya la decisión de Espina.

${ }^{11}$ El depuesto presidente Serrano se retiró de Guatemala, teniendo como primer destino El Salvador. Durante su estada en este pais manifestó que al dar el autogolpe había cavado su tumba política. $L a$ Nación, Buenos Aires, 6 de junio de 1993. Con posterioridad, Serrano pidió asilo político en Panamá. 
En la Plaza Mayor se realiza una nueva manifestación popular exigiendo la inmediata renuncia de Espina y rechazando el nuevo gobiemo de facto.

La Corte Suprema de Justicia envía al Congreso Nacional la solicitud de antejuicio contra Serrano y Espina, planteada por el Ministerio Público.

Los tribunales de justicia ordenan el arraigo de los magistrados que asumieron sus cargos en la Corte Suprema de Justicia nombrada ilegalmente por Serrano, a fin de iniciar un proceso judicial en su contra.

Ante estas instancias judiciales, Espina advierte sobre la posibilidad de presentar recursos de amparo contra la Corte de Constitucionalidad y contra el juez que inició proceso en su contra, argumentando que tiene inmunidad por el cargo que ocupa.

El día 3 de junio, hombres armados llegan al Ministerio Público, buscando al jefe de dicha repartición, Edgar Tuna Valladares, promotor de los antejuicios enunciados.

A solicitud de Espina renuncian todos los ministros con excepción del Ministro de Defensa y de la Gobemación.

Se produce un paro nacional de los empleados de la Administración Pública, desconociendo la autoridad de Espina como Jefe del Gobiemo y solicitando la reinstalación del régimen institucional.

El 4 de junio, la Corte de Constitucionalidad hace conocer un dictamen sobre las normas temporales del gobierno, según el cual Espina está inhabilitado para ocupar la Presidencia de Guatemala, como lo estaba Serrano, y declara vacante el cargo de Presidente.

En el mismo dictamen se establece que el Congreso Nacional tiene un plazo de 24 horas para elegir, de acuerdo a la Constitución Nacional, el nuevo Presidente para completar el periodo que finaliza el 14 de enero de 1996. De igual manera, se determina que, en su oportunidad, se elija al Vicepresidente de la República.

Los acontecimientos provocaron la renuncia de Espina, quien declara que "como Vicepresidente de la República, popularmente electo en los comicios del 6 de enero de 1991, me correspondía, de acuerdo al articulo 189 de la Constitución, asumir el cargo de Presidente de la República. En aras de la concordia nacional y con el anhelo de contribuir a que el país vuelva a la institucionalidad, presento mi renuncia irrevocable al cargo de Vicepresidente de la República". ${ }^{12}$

\footnotetext{
${ }^{12}$ El Vicepresidente Espina pidió asilo diplomático en Costa Rica. Una vez normalizada la situación de inestabilidad política con la presidencia de León Carpio, el Ministro de Relaciones Exteriores, Arturo Fajardo, solicitó a los gobiernos de Panamá y Costa Rica la extradición de Serrano y Espina, dando trámite a una solicitud de la Fiscalia que acusa a los mencionados funcionarios de cometer delitos, entre los que se incluyen violación de la Constitución, enriquecimiento ilicito y usurpación de poderes. Clarin, Buenos Aires, 20 de junio de 1993).
} 
El 5 de junio, el Congreso Nacional designa como Presidente de Guatemala al licenciado Ramiro de León Carpio. ${ }^{13}$

El 18 de junio fue electo Vicepresidente de la República Arturo Herbruger Asturias, ex-Presidente del Tribunal Supremo Electoral. ${ }^{14}$

De esta manera, se pudo superar mediante una intrincada red de actores nacionales, el segundo y tercer autogolpe producido en un mismo país de América en la presente década.

\section{Consideraciones finales.}

Es prácticamente imposible en un trabajo de estas caracteristicas dedicarse a profundizar el papel que le tocó desempeñar a cada uno de los grupos o actores intemos que favorecieron la instalación del gobierno de facto de Serrano o de los que impidieron la consolidación del autogolpe. ${ }^{15}$

Pero coincido con Rigoberta Menchú en que las tendencias contrarias al golpe de Estado se estaban desarrollando en la arena política informal de la sociedad civil, fuera del sistema político formal. ${ }^{16}$

De cualquier manera, se debe establecer la distinción entre aquellas asociaciones civiles que presionaron para evitar la ruptura constitucional y las instituciones que encauzaron esas presiones o protestas.

\footnotetext{
${ }^{13}$ Los candidatos para ocupar la Presidencia de la República fueron: Arturo Herbruger Asturias, Presidente del Tribunal Supremo Electoral; Ramiro de León Carpio, Procurador de Derechos Humanos; Epaminondas González Durbón, Presidente de la Corte de Constitucionalidad; Jorge Mario Garcia Laguardia, Magistrado de la Corte de Constitucionalidad; Edmundo Vásquez Martinez, ex-Presidente de la Corte Suprema de Justicia; Fernando Andrade Diaz-Durán, ex-Canciller, Mario Quiñórez Amézquita, ex-Canciller, Manuel Ayau, dirigente empresarial; y Rigoberta Menchú Tum, Premio Nobel de la Paz. Luego de intensas conversaciones y negociaciones, a solicitud de la Instancia de Consenso Nacional, la nómina de candidatos se redujo a dos: Ramiro de León Carpio obtiene 64 votos y Herbruger 51 , no logrando el primero los dos tercios necesarios exigidos por la ley. Al conocerse este resultado, Arturo Herbruger Asturias declina su candidatura en aras de la concordia nacional y de la superación del impasse por el que atraviesa Guatemala. En la segunda votación, de León Carpio obtiene 106 volos, sobre una totalidad de 115 presentes. Como se puede observar, 9 legisladores rehusaron darle su voto, y un diputado estuvo ausente.

${ }^{14}$ De acuerdo a la Constitución Nacional, el Congreso debe elegir al Vicepresidente en base a una terna propuesta por el Presidente de la nación. I a terna estaba compuesta por Arturo Herbruger Asturias, quien obtuvo 101 yotos; Carlos Garcia Bauer, 8 votos; y Gustavo Hernández Polanco con un voto. No asistieron a la sesión seis diputados.

15 Sería injusto no mencionar la activa participación en la sociedad guatemalteca de los grupos que aportaron al restablecimiento democrático. Entre ellos podemos mencionar a los medios de comunicación, la Instancia Nacional de Consenso, los empresarios, estudiantes, sindicatos, Iglesia Católica, partidos políticos y cuerpo diplomático.

${ }^{16}$ Jonas, Susanne, op. cit., p. 17.
} 
Es por ello que me pareció pertinente a los fines pedagógicos, resaltar o privilegiar desde el punto de vista interno el rol que desempeñó la justicia en la solución de la crisis.

En este sentido me refiero a la participación de la Corte de Constitucionalidad, a la Procaduría de Derechos Humanos y al Tribunal Superior Electoral.

Como pudimos observar, la rápida y oportuna reacción de la Corte de Constitucionalidad representó el primer hito para evitar la consolidación del régimen de facto.

La actuación de la Corte de Constitucionalidad mostró su efectividad no sólo en el primer golpe de Estado de Serrano, sino también en el segundo golpe de Espina.

La decisión de las fuerzas armadas para exigir la renuncia de Serrano y Espina se basó en la resolución de esta Corte, del 25 de mayo, ratificada por el dictamen del 4 de junio.

Debe mencionarse también el papel destacado que le cupo a la Procaduría de Derechos Humanos.

El mismo día del golpe de Estado, al procurador de los Derechos Humanos, Ramiro de León Carpio -quien había sido designado para ese cargo por el Congreso Nacional-, se le notifica que pesaba sobre su persona un arresto domiciliario.

En esa ocasión, escapando del arresto y acompañado por los embajadores de la Comunidad Europea, que condenaron el régimen impuesto, manifestó: "Me suspendo yo mismo de mis funciones". Esta suspensión duraría, según de León Carpio, hasta tanto se restaurara la Constitución. ${ }^{17}$ De León Carpio fue electo Presidente por el Congreso Nacional luego de la renuncia de Espina.

El tercer hito significativo por parte de la justicia, estuvo a cargo del Tribunal Superior Electoral. Este informó que no convocaría ni a elección de una asamblea constituyente ni a un referéndum hasta que la Constitución no fuera restablecida por completo.

El Presidente del Tribunal Superior Electoral, Arturo Herbruger, dijo que "cualquier consulta electoral debe llevarse a cabo en una situación de plenas garantías". 18

\footnotetext{
17 "Abierta condena al autogolpe de Serrano", La Capital, Rosario, 27 de mayo de 1993, p. 3.

${ }^{18}$ No puede dejar de mencionarse que esta decision del Tribunal Superior Electoral fue adoptada por unanimidad de los cinco miembros integrantes. La independencia de este Tribunal del poder político, quedó demostrada luego de organizar y supervisar cinco elecciones desde 1985, sin que se registrase ninguna denuncia de fraude. Roberts, Carlos Reymundo, "Serrano no logra consolidar su poder", La Nación, Buenos Aires, 31 de mayo de 1993, p. 3.
} 
Esta postura del Tribunal Superior Electoral, puso punto final a las pretensiones de Serrano de legitimar el golpe de Estado a través de las umas, como en su oportunidad lo había hecho el Presidente peruano Fujimori en 1992.

En una comparación con el golpe de Estado en Perú, se puede apreciar que en este caso, Serrano el mismo día del golpe, anunció la elección de una asamblea constituyente. En el caso peruano, la decisión de Fujimori nació a partir de las presiones externas, sobre todo de la Organización de Estados Americanos (OEA) y del gobierno de los Estados Unidos, con posterioridad al autogolpe.

Si bien en una situación de esta naturaleza (el autogolpe del 25 de mayo de 1993), no se puede asegurar con certeza que exista un único factor que impida la consolidación dé un golpe de Estado, es indudable que la justicia, con excepción de la Corte Suprema de Justicia, cumplió en Guatemala un rol significativo.

A nivel internacional, debe hacer la misma distinción que se produjo a nivel doméstico. Las manifestaciones de rechazo al golpe de Estado por parte de numerosos países, que se evidenció en hechos concretos, así como la suspensión de ayuda económica inmediata, lograron crear un clima internacional desfavorable para las aspiraciones de Serrano de continuar en el poder.

A esta presión internacional se sumó una misión de la Organización de Estados Americanos encabezada por el Secretario General, Joao Baena Soares, que por medio de la negociación logró marginar a Serrano y apresurar el retorno de la democracia.

Es por ello que en el presente trabajo se hace hincapié en la situación de la OEA, sin dejar de resaltar el accionar de países, organismos internacionales gubernamentales y no gubernamentales, que propiciaron la ayuda necesaria para abortar esta nueva experiencia de autogolpe en América Latina. ${ }^{19}$

Los acontecimientos de Guatemala llamaron la atención de la Organización de Estados Americanos que desde 1991 se había preocupado de

\footnotetext{
${ }^{19}$ Entre los paises que manifestaron su oposición al golpe de Estado se puede citar entre otros: Alemania, Argentina, Brasil, Costa Rica, Chile, España, Estados Unidos de América, Italia, Japón, Nicaragua y' Venezuela. Se debe mencionar que algunos paises apoyaron al golpe de Estado, como fue el caso de Perú. En el ámbito de los organismos internacionales gubernamentales, se puede incluir entre otros a la Organización de las Naciones Unidas, la Comunidad Europea y el Grupo de Río, si bien este último no tiene las características propias de los organismos internacionales y actúa como mecanismo de consulta y concertación. Entre los organismos no gubernamentales que demosiraron su repudio al golpe, se pueden citar el Parlamento Latinoamericano, la Internacional Demócrata Cristiana, la Federación Internacional de Periodistas (F.I.P.) y Amnesty Internacional.
} 
prevenir los golpes de Estado en la región a través de resoluciones y declaraciones.

Es así que el mismo día del golpe de Estado emite una declaración en Washington manifestando su preocupación por los acontecimientos de Guatemala. Se decide, así mismo, enviar una misión encabezada por el Secretario General de la OEA, Joao Baena Soares y tres ministros de Relaciones Exteriores para evaluar in situ la inestabilidad institucional.

Simultáneamente, convoca con urgencia a los embajadores para estudiar la situación y las medidas por adoptar.

De acuerdo al mandato de la OEA, el 29 de mayo, arriba la misión de Guatemala. En esa ocasión, el Secretario General expresó: "Vamos a reunimos con el señor Presidente de la nación, con las autoridades y los jefes de la sociedad guatemalteca para escuchar sus opiniones" 20

Entre las rcuniones realizadas por la misión de la OEA en Guatemala, merecen destacarse dos. Una efectuada con el Presidente de la nación y otra con los altos mandos de las fuerzas armadas.

En una audiencia con el Presidente, el sábado 29 de mayo, el Canciller de Uruguay, Sergio Abreu Bonilla, quien integraba la delegación de la OEA, preguntó a Serrano si estaba dispuesto a un renunciamiento personal para superar la crisis.

El Presidente Serrano manifestó que no había considerado la posibilidad, pero respondió afirmativamente agregando como condiciones no ser "perseguido políticamente después" y que se le dejara abierta la puerta para volver a ser candidato, mediante una reforma de la Constitución.

La otra reunión importante mantenida por la OEA en suelo guatemalteco, se efectuó con los militares. A la misma concurrieron los generales José Domingo Garcia Samoyoa (Ministro de Defensa), Francisco Ortega Manaldo (Jefe del Estado Mayor de la Presidencia de la Nación), Otto Pérez Molina (Inteligencia), Mario Enríquez (Miembro de la Comisión de Paz) y Roberto Perussina (Jefe del Estado Mayor).

De acuerdo a Cardoso, los militares se limitaron a escuchar los argumentos de la OEA. La cúpula militar escuchó en silencio al Secretario General de la OEA, Joao Baená Soares, quien expresó que para el organismo interamericano, el golpe de Serrano no es un Fujimori II. La situación de Guatemala no es equiparable a la de Perú.

\footnotetext{
${ }^{20}$ Para el seguimiento de la actuación de la OEA en la crisis, se recurrió a documentos de esa organización y al relato de periodistas destacados en Guatemala. Oscar Raúl Cardoso, "Las FF.AA. y la oposición negocian la destitución del Presidente de Guatemala", Clarin, Buenos Aires, 1 de junio de 1993, p. 32/33; y Carlos Reymundo Roberts, "Un Presidente en la cornisa", La Nación, Buenos Aires, I de junio de 1993, p. 4.
} 
El Secretario General manifestó que no existe por parte de la OEA la más mínima voluntad de hacer concesiones o negociaciones con Serrano. La única solución aceptable para el Secretario General era el retorno a la más plena legalidad constitucional. La Organización también sostiene que no se convalidarán esquemas de maquillaje que oculten la ruptura democrática y que si Guatemala no restablece el orden institucional deberá enfrentar las sanciones políticas y económicas que se propongan en la reunión de los cancilleres convocada por la OEA.

Existe coincidencia de que fue inmediatamente después de esta entrevista que el Ministro de Defensa, García Samayoa, formuló la frase que precipitaría el destino de Serrano. En esta ocasión el Ministro dijo: "Hay que volver a la normalidad constitucional lo antes posible".

La misión de la OEA fue el factor determinante en la resolución de la crisis que concluyó con la destitución de Serrano.

La acción de la justicia a nivel interno como cauce a las expresiones de rechazo a los autogolpes de Guatemala y el poder de negociación de la Organización de Estados Americanos a nivel intemacional, se conjugaron para poder evitar la repetición de otro autogolpe de Estado en América Latina.

Es por ello que creo importante como ejemplo para el resto de la región dedicar este trabajo al caso Guatemala, para poder apreciar cuáles son las fuerzas internas y externas que pueden impedir los golpes de Estado en el futuro institucional de América Latina como área democrática. 